\title{
State-Dependent Release of Acetylcholine in Rat Thalamus Measured by in vivo Microdialysis
}

\author{
Julie A. Williams, Jeff Comisarow, Jamie Day, Hans C. Fibiger, and Peter B. Reiner \\ Kinsmen Laboratory of Neurological Research, Department of Psychiatry, University of British Columbia, Vancouver, \\ Canada V6T 1 Z3
}

\begin{abstract}
Mesopontine cholinergic neurons have long been thought to play a key role in behavioral state control. In particular, they have been implicated in the process of EEG desynchrony and in the generation of rapid eye movement (REM) sleep. However, the behavioral profile of identified mesopontine cholinergic neurons has not been unequivocally demonstrated. In an attempt to address this issue, in vivo microdialysis was used to monitor acetylcholine (ACh) release across behavioral state in the rat thalamus, a major projection site of mesopontine cholinergic neurons. Because REM periods in rats are of short duration, a method was developed to collect and accumulate sufficiently large samples from each of the individual states of wake, slow-wave sleep, and REM sleep to permit off-line analysis via (HPLC-ECD). Probe placement and the source of cholinergic innervation to the vicinity of the microdialysis probe were verified using retrograde tracing combined with ChAT immunohistochemistry. Finally, the sodium and calcium dependence of $\mathrm{ACh}$ measured in the thalamus were tested using $\mathrm{TTX}$ and calcium-free dialysates. The results showed that (1) extracellular ACh concentrations in the thalamus are high during both wake and REM sleep and significantly lower during slow-wave sleep, (2) the majority of cholinergic projections to the vicinity of the dialysis probes originate in the mesopontine tegmentum, and (3) ACh release in the thalamus is due to sodium- and calcium-dependent mechanisms. In contrast to predictions of some previous hypotheses, these results demonstrate that mesopontine cholinergic neurons are active during both wake and REM sleep.
\end{abstract}

[Key words: ACh, mesopontine cholinergic neurons, REM sleep, behavioral state control, EEG, microdialysis]

Mesopontine cholinergic neurons are thought to have important roles in EEG desynchrony and in the generation of rapid eye movement (REM) sleep. This notion has been supported by a large body of work originating with Moruzzi and Magoun (1949), who found that eliciting EEG desynchrony by electrical stimulation in the brainstem was most effective when the stimulating electrode was placed in the region of the mesopontine tegmen-

\footnotetext{
Received Nov. 29, 1993; revised Feb. 7, 1994; accepted Feb. 24, 1994.

We thank Dr. John Pinel for generously offering the use of his polygraph; Catriona Wilson, Chui-Se Tham, and Andrew Laycock for superb technical assistance; and Dr. Campbell Clarke for assisting with the statistical analyses. This work was supported by grants from the MRC (MT-10399, PG-23). P.B.R. is an MRC Scientist. J.A.W. and J.D. arc supported by MRC studentships.

Correspondence should be addressed to Peter B. Reiner, Kinsmen Laboratory of Neurological Research, Department of Psychiatry, University of British Columbia, 2255 Wesbrook Mall, Vancouver, B.C. Canada V6T 1Z3.

Copyright (C) 1994 Society for Neuroscience $0270-6474 / 94 / 145236-07 \$ 05.00 / 0$
}

tum. Early brainstem lesion studies showed that lesions at the midbrain-pontine junction abolished EEG desynchrony during wake (Lindsley et al., 1949, 1950; Batini et al., 1959; Jouvet, 1962; Hobson, 1965), and reduced the phasic activity normally observed during REM sleep, including rapid eye movements, PGO waves, and muscle atonia (Jouvet, 1962; Hobson, 1965). It was later shown by means of acetylcholinesterase (AChE) histochemistry and from choline acetyltransferase (ChAT) immunohistochemistry that the brainstem cholinergic neurons are localized in the laterodorsal and pedunculopontine tegmenta (LDT, PPT, respectively; Shute and Lewis, 1967; Satoh et al., 1983; Jones and Beaudet, 1987; Vincent and Reiner, 1987). In addition, retrograde and anterograde tracing combined with ChAT immunohistochemistry revealed that mesopontine cholinergic neurons gave rise to a massive innervation of the thalamus in rats (Sofroniew et al., 1985; Satoh and Fibiger, 1986; Woolf and Butcher, 1986; Hallanger and Wainer, 1988; Semba and Fibiger, 1992), which is thought to control cortical EEG (Steriade et al., 1990a). Mesopontine cholinergic neurons thus became one of the neuronal candidates for Moruzzi and Magoun's "ascending reticular activating system" (1949).

Recent brainstem lesion studies cmploying modern techniques to further localize damage to the LDT/PPT have suggested that mesopontine cholinergic neurons may be crucial to the generation of REM sleep and its associated features. Shouse and Siegel (1992) found that lesion of the PPT reduces the amount of PGO activity in REM sleep. Webster and Jones (1988) demonstrated that lesion of the LDT/PPT cholinergic neurons eliminated REM sleep in some animals for up to several weeks, while in others, PGO activity and muscle atonia during REM sleep were reduced - the extent of the reduction of time spent in REM sleep and of PGO activity correlated with the extent of the loss of cholinergic neurons. Unlike earlier studies, in which lesions encompassed a much larger area (Lindsley et al., 1949, 1950; Batini et al., 1959; Jouvet, 1962; Hobson, 1965), waking and EEG desynchrony were not significantly altered, which suggested that other neuronal substrates were involved in the control of waking EEG.

The importance of LDT/PPT cholinergic neurons in the generation of REM sleep is further supported by the observation that microinjection of the cholinergic agonist carbachol into the medial pontine reticular formation (mPRF) induces a state that is indistinguishable from REM sleep (George et al., 1964; Baxter, 1969; Mitler and Dement, 1974; Amatruda et al., 1975; Gnadt and Pegram, 1986). Anatomical data have shown that the MPRF is innervated by cholinergic neurons originating in the LDT/PPT (Mitani et al., 1988; Shiromani et al., 1988; Quatrocchi et al., 1989; Jones, 1990; Semba et al., 1990; Semba, 
1993). It has therefore been hypothesized that release of $\mathrm{ACh}$ in the MPRF is perhaps the natural trigger for REM sleep.

The interaction of cholinergic, noradrenergic, and serotonergic neurons in the brainstem has long been suggested to be a key factor in behavioral state control (Hernandez-Peon, 1963; Jouvet, 1969; Hobson et al., 1975). That amines inhibit AChinduced triggering of REM sleep was first suggested by the observation that systemic injection of the AChE inhibitor eserine induced a state that was indistinguishable from REM sleep, but only after depletion of amines with reserpine (Karczmar et al., 1970). Electrophysiological rccordings in the locus ceruleus and in the dorsal raphe showed that noradrenergic and serotonergic neurons are very active during wake, less so during slow-wave sleep (SWS), and silent during REM sleep (Hobson et al., 1975; McGinty and Harper, 1976; Trulson and Jacobs, 1979; AstonJones and Bloom, 1981; Reiner, 1985). Based upon these findings, it was hypothesized that amines have an inhibitory influence on brainstem cholinergic mechanisms (Karczmar et al., 1970; McCarley and Hobson, 1975; Sakai, 1988; Steriade and McCarley, 1990). Indeed, recent studies have shown that noradrenaline (NA) and serotonin (5-HT) inhibit the majority of rat LDT neurons in vivo (Koyama and Kayama, 1993) as well as hyperpolarize identified mesopontine cholinergic neurons in brainstem slices in vitro (Luebke et al., 1992; Williams and Reiner, 1993). Taken together, these data suggest that brainstem cholinergic neurons would be powerfully inhibited during wake, when both serotonergic and noradrenergic neurons are most active.

On the other hand, while the data reviewed above may suggest that mesopontine cholinergic neurons are REM specific, in vivo electrophysiological recordings in the mesopontine tegmentum have revealed that only a minority of cells fire selectively during REM sleep, and that a majority fire both during REM sleep and wake (Mansari et al., 1989; Steriade et al., 1990b; El Kayama et al., 1992). Thus, the state-dependent activity of identified mesopontine cholinergic neurons remains unclear. In an attempt to resolve this issue, we carried out experiments using in vivo microdialysis, in which $\mathrm{ACh}$ release was measured across behavioral state in the rat thalamus. Several anatomical investigations have shown that the rat thalamus receives cholinergic projections exclusively from the brainstem, with the exception of the reticular nucleus, which receives cholinergic innervation from both the basal forebrain as well as the mesopontine tegmentum (Sofroniew et al., 1985; Satoh and Fibiger, 1986; Woolf and Butcher, 1986; Hallanger and Wainer, 1988; Jourdain et al., 1989). The medial portion of the thalamus, therefore, is an ideal region to test the extent to which there is state-dependent $\mathrm{ACh}$ release from brainstem cholinergic neurons.

\section{Materials and Methods}

Surgery. Male wistar rats, weighing 275-390 gm, were surgically implanted with EEG recording electrodes for monitoring behavioral state and with a transverse microdialysis probe in the thalamus for measuring $\mathrm{ACh}$ concentrations in vivo.

Animals were anesthetized with $50-60 \mathrm{mg} / \mathrm{kg}$ pentobarbital (i.p.) and supplemented as needed. For EEG recordings, animals were chronically implanted with three screw electrodes (two for cortical EEG and one over the cerebellum as a reference) and with either nuchal electrodes for EMG or depth electrodes for the recording of hippocampal theta (AP -3.1, DV -3.0, ML - 2.4 from bregma; Paxinos and Watson, 1982). Electrode pins were held in place with an Amphenol strip connector and fixed to the skull with dental acrylic.

Transverse microdialysis probes were constructed and implanted as described in detail elsewhere (Damsma and Westerink, 1991). Briefly, probes were made of a Hospal Filtral AN69 microdialysis membrane ( $0.22 \mathrm{~mm}$ i.d., $0.27 \mathrm{~mm}$ o.d., 60,000 molecular weight cutoff) with an active surface of $7 \mathrm{~mm}$. Dialysis probes were placed at stereotaxic coordinates from bregma (AP $-3.3, \mathrm{DV}-5.8$ to -5.6 ; Paxinos and Watson, 1982), and the active surface centered to expose the ventroposterolateral, ventroposteromedial, centromedial, mediodorsal, interomediodorsal, and posterior thalamic nuclei.

Microdialysis. Following surgery, rats were housed individually in 35 $\times 35 \times 25 \mathrm{~cm}$ Plexiglas cages and were given food and water ad libitum. On the first day after surgery, each animal was moved into a secluded recording room, where they were attached to a model 78 Grass polygraph for several hours for adaptation. Experiments were performed on the second and third days after surgery during the light phase of a 12: $12 \mathrm{hr}$ light:dark cycle (from 0800 to $2000 \mathrm{hr}$ ) between 1000 and 1900 $\mathrm{hr}$. Because the different behavioral states of the rat are short lived, the sample collection procedure (Damsma and Westerink, 1991) was modified in order to achieve the purest dialysis samples possible for each of the behavioral states of wake, SWS, and REM sleep. The samples were collected as follows. The dialysis probes were perfused with artificial cerebrospinal fluid (ACSF) containing (in $\mathrm{mM}$ ) $125 \mathrm{NaCl}, 3 \mathrm{KCl}$, $1.3 \mathrm{CaCl}_{2}, 1.0 \mathrm{MgCl}_{2}, 23 \mathrm{NaHCO}_{3}, 1.0 \mathrm{PO}_{4}$ buffer, and $\mathrm{pH}$ balanced to 7.4 with $\mathrm{HCl}$. In order to recover detectable levels of extracellular $\mathrm{ACh}$, the reversible $\mathrm{AChE}$ inhibitor neostigmine bromide (Sigma) was added to the ACSF (100 nM). The dialysis membrane was perfused at a rate of $5 \mu \mathrm{l} / \mathrm{min}$, controlled by a syringe pump (Harvard Apparatus). The probe inlet was connected to the syringe by polyethylene tubing $(800$ $\mathrm{cm} \times 0.28 \mathrm{~mm} ; 50 \mu \mathrm{l}$ in volume). The outlet polyethylene tubing was also cut to a $50 \mu \mathrm{l}$ volume length and weighted at the end with a fitted short piece of Tygon tubing.

For the behavioral experiments, all dialysate samples werc collected into ice-cold microcentrifuge tubes marked "wake," "SWS," or "REM." Each vial contained $5 \mu \mathrm{l}$ of $0.4 \%$ acetic acid in order to stabilize the ACh collected in the dialysate. No more than $100 \mu \mathrm{l}$ of dialysate was allowed to accumulate in each vial in order to maintain equal concentrations of acetic acid for each of the behavioral states. As the animals shifted from wake to SWS to REM, each state was timed and a $10 \mathrm{~min}$ $30 \mathrm{sec}$ delay was added to allow the perfusate to move from the animal to the end of the output tubing.

Samples that were collected in the "wake" vials included periods in which the animal was clearly alert with a desynchronized EEG and engaged in some sort of waking behavior such as grooming, eating, drinking, or exploring. "SWS" vials included periods in which the animal was in a sleeping position, eyes closed, and the EEG synchronized for $>30 \mathrm{sec}$ at a time. "REM" vials included the sleep state in which the animal exhibited muscle twitches, EEG desynchrony, and theta activity. All samples from ambiguous and transition states were discarded.

Prior to sample collection, animals were dialyzed for $40-60 \mathrm{~min}$ in order to allow the brain to equilibrate with the perfusion solution. Samples were collected during 5-6 hr sessions and were stored in an ice bucket for off-line analysis via high-performance liquid chromatography in conjunction with electrochemical detection (HPLC-ECD) immediately after the session.

To test for the sodium and calcium dependence of thalamic ACh release, animals were attached directly to the HPLC system for on-line analysis of $\mathrm{ACh}$ release. Once steady baseline levels were achieved, animals were perfused either with solution containing $1 \mu \mathrm{M}$ tetrodotoxin (TTX; Sigma) or with calcium-free perfusate for at least $30 \mathrm{~min}$.

Assay of acety/choline. For the off-line experiments, dialysis samples were manually injected into a $50 \mu$ sample loop of the analytical system with a $100 \mu \mathrm{l}$ Hamilton syringe. For the on-line experiments, the output tubing was connected directly to the sample loop, and perfusate was injected at $10 \mathrm{~min}$ intervals controlled by an adjustable timer (Valco). Standards $\left(10^{-7} \mathrm{M} \mathrm{ACh}\right)$ were injected hourly into a separate $20 \mu \mathrm{l}$ sample loop.

The assay of ACh via HPLC-ECD was performed in conjunction with an enzyme reactor (Damsma et al., 1987). Briefly, after separation of $\mathrm{ACh}$ and choline $(\mathrm{Ch})$ with a reverse phase column $(75 \times 2.1 \mathrm{~mm})$ pretreated with lauryl sulfate, the eluent was then passed through an enzyme reactor $(10 \times 2.1 \mathrm{~mm})$ containing acetylcholinesterase (EC 3.1.1.7; Sigma, type VI-S) and choline oxidase (1.1.3.17; Sigma) covalently bound to glutaraldehyde-activatcd Lichrosorb $\mathrm{NH}_{2}(10 \mu \mathrm{m}$; Merck). The reaction of $\mathrm{ACh}$ and $\mathrm{Ch}$ with these enzymes produced a quantitative yield of hydrogen peroxide, which was electrochemically detected at a platinum working electrode set at a potential of $+500 \mathrm{mV}$ 
Table 1. Extracellular ACh concentrations in the thalamus across behavioral state

\begin{tabular}{clcl} 
Rat No. & Wake & SWS & REM \\
\hline 3 & $14.73(0.47)$ & $7.87(0.75)$ & 16.4 \\
4 & $10.53(3.0)$ & $7.00(0.7)$ & $10.36(0.36)$ \\
& $11.09(1.70)$ & $10.39(1.27)$ & $18.91(0.73)$ \\
5 & $8.10(1.22)$ & $5.17(0.69)$ & $10.00(1.00)$ \\
& $8.13(1.30)$ & $6.00(0.39)$ & $11.56(0.88)$ \\
8 & $19.19(3.61)$ & $13.66(1.66)$ & 22.99 \\
& $15.19(1.38)$ & $9.17(1.13)$ & 12.90 \\
10 & $13.49(1.23)$ & $9.04(0.37)$ & 18.07 \\
& $7.83(0.79)$ & $4.35(0.26)$ & 7.68 \\
15 & $21.74(0.31)$ & $8.40(0.59)$ & 23.94 \\
& $24.23(0.67)$ & $6.69(1.72)$ & $20.53(0.52)$ \\
18 & $35.57(0.60)$ & $8.89(1.00)$ & 30.27 \\
26 & $18.93(0.89)$ & $4.97(0.58)$ & $14.91(1.42)$ \\
31 & 31.37 & $9.59(0.62)$ & 37.91 \\
32 & $30.06(1.71)$ & $11.28(0.37)$ & 36.36
\end{tabular}

Mean ( \pm SD) ACh concentrations are reported as $\mathrm{fmol} / \mathrm{min}$ for each behavioral state in individual rats.

versus an $\mathrm{Ag} / \mathrm{AgCl}$ reference electrode (BAS-LC4B or ANTEC VT-03). An HPLC pump (LKB-2150 or Shimadzu LC-600) delivered the mobile phase $(0.2 \mathrm{M}$ aqueous potassium phosphate buffer $\mathrm{pH} 8.0,1 \mathrm{~mm}$ tetramethylammonium hydroxide) at a rate of $0.4 \mathrm{ml} / \mathrm{min}$. Chromatograms were drawn on a chart recorder (Kipp); hydrogen peroxide pcaks corresponding to ACh normally appeared $4-5 \mathrm{~min}$ after the initial solvent front. The detection limit of the assay was $50 \mathrm{fmol}$ per injection.

Immunohistochemistry. Probe placement was verified in four rats using standard techniques, while in six rats, the source of cholinergic innervation to the probe was also verified using Fluoro-Gold (FG) as a retrograde tracer ( $4 \%$ in $0.9 \%$ saline) and ChAT immunohistochemistry. FG was manually injected into the dialysis probe and rinsed out with normal perfusion solution after $24 \mathrm{hr}$. After 5-14 d, animals were anesthetized with an overdose of sodium pentobarbital and transcardially perfused with $100 \mathrm{ml}$ of $0.9 \%$ saline followed by $300 \mathrm{ml}$ of $4 \%$ paraformaldehyde in $0.1 \mathrm{M}$ phosphate buffered saline (PBS). The brains were removed and postfixed at $4^{\circ} \mathrm{C}$ for $2 \mathrm{hr}$ and then transferred to $15 \%$ sucrose in $0.1 \mathrm{M}$ PBS and stored overnight at $4^{\circ} \mathrm{C}$. The next day, brains were cut into $30 \mu \mathrm{m}$ coronal sections on a freezing microtomc and cvery fifth section separated into wells containing $0.1 \mathrm{M}$ PBS.

Sections that contained the site of the probe were mounted onto coated slides immediately for verification of placement. Ten cortical sections containing basal forebrain cholinergic neurons, and 10 brainstem sections containing LDT/PPT cholinergic neurons were selected and processed for ChAT immunohistochemical staining as follows. The 20 sections were placed in a solution composed of 1:50 normal goat serum (Jackson Immunoresearch Laboratories, Inc.), 0.02\% sodium azide, and 1:50 rat anti-ChAT antibody (Boehringer-Mannheim) in 0.1 M PBS containing $0.3 \%$ Triton and incubated for 2 nights at $4{ }^{\circ} \mathrm{C}$. Sections were washed $3 \times 20 \mathrm{~min}$ in PBS and then incubated at room temperature for $2 \mathrm{hr}$ in 1:20 Texas red-conjugated goat anti-rat antibody (Jackson Immunoresearch Laboratories, Inc.) in $0.3 \%$ Triton/PBS. After the 2 hr period, slices were washed again for $3 \times 20 \mathrm{~min}$ in $0.1 \mathrm{M}$ PBS and mounted onto coated slides for inspection using fluorescence microscopy.

For identifying and quantifying double-labeled cells, basal forebrain and brainstem sections were photographed twice: once using green light $(530-560 \mathrm{~nm})$ to visualize the Texas red-labeled cholinergic neurons, and once using ultraviolet light $(340-380 \mathrm{~nm})$ to visualize the retrogradely labeled cells. Negatives obtained under the green light were projected onto $8.5 \times 11$ inch sheets of paper, onto which the individual cholinergic cells were traced. Afterward, the corresponding exposures under ultraviolet fluorescence were projected onto the drawing for identification of double-labeled cells by the superimposed images of the retrogradely labeled neurons.

Statistical analyses. One-way analysis of variance (ANOVA) with repeated measures was used to analyze the effect of bchavioral state on

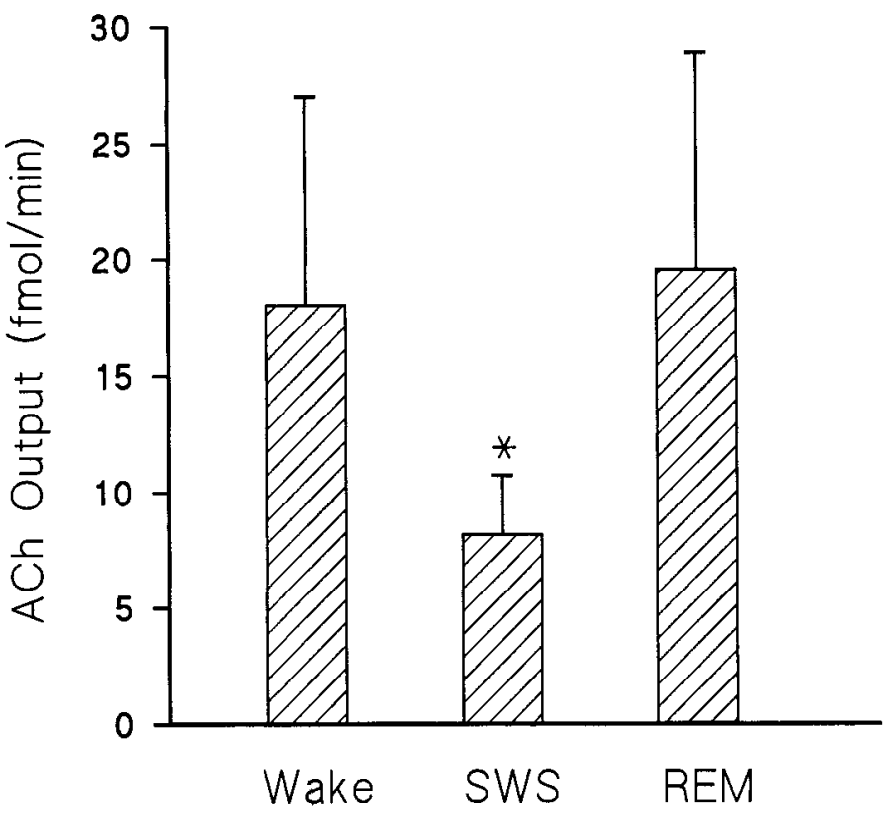

Figure 1. Extracellular acetylcholine concentrations across behavioral state in the rat thalamus. Mean ACh concentrations ( $\pm \mathrm{SD}$, error bars) are reported for wake, slow-wave sleep (SWS), and REM sleep $(n=15$ for each behavioral state). ACh release was significantly higher in REM sleep and wake than in SWS $(p<0.0001)$.

ACh concentrations. Scheffe's test was used for the comparison of the ACh concentrations between each of the states. Student's $t$ test was used to analyze the effect of 'TIX and calcium-free perfusate on ACh release. $A C h$ concentrations are reported as femtomoles per minute $(\mathrm{fmol} / \mathrm{min}$ ).

\section{Results}

\section{Thalamic acetylcholine release}

In order to determine whether $\mathrm{ACh}$ release in the thalamus was the result of action potential-dependent neuronal activity, we carried out two experiments. First, TTX $(1 \mu \mathrm{M})$ was added to the perfusion solution to block voltage-dependent sodium channels. TTX significantly reduced extracellular ACh concentrations in two of two rats from an average baseline of $18.21 \pm$ 5.82 to $2.12 \pm 1.31 \mathrm{fmol} / \mathrm{min}(t=7.62, p<0.0001)$. Second, two other rats were perfused with calcium-free ACSF in order to abolish $\mathrm{Ca}^{2+}$-dependent synaptic transmission. The calciumfree solution significantly reduced the baseline ACh concentrations in two of two rats from $15.75 \pm 7.25$ to $3.51 \pm 0.75 \mathrm{fmol} /$ $\min (t=5.57, p<0.0001)$. Thesc rcsults confirm that thalamic ACh release was due to sodium- and calcium-dependent mechanisms, as opposed to overflow of extracellular neurotransmitter caused by trauma from the probe implant (Westerink and De Vries, 1988).

The number of $50 \mu \mathrm{l}$ samples obtained on any particular day for each rat varied between the behavioral states of wake, SWS, and REM. An average of $4.93 \pm 2.72$ samples were obtained for wake, $5.13 \pm 2.00$ for SWS, and $1.53 \pm 0.50$ for REM on each day. Because most of the samples within each state for each rat were very similar, within-state samples were averaged for each experimental day. A total of 15 sample values for each behavioral state were obtained in 10 rats. These values and their standard deviations are reported in Table 1.

The average $\mathrm{ACh}$ concentration during wake was $18.01 \pm$ $8.58 \mathrm{fmol} / \mathrm{min}$; during SWS, $8.17 \pm 2.55 \mathrm{fmol} / \mathrm{min}$; and during REM, $19.52 \pm 9.36 \mathrm{fmol} / \mathrm{min}$ (Fig. 1). Analysis of variance 

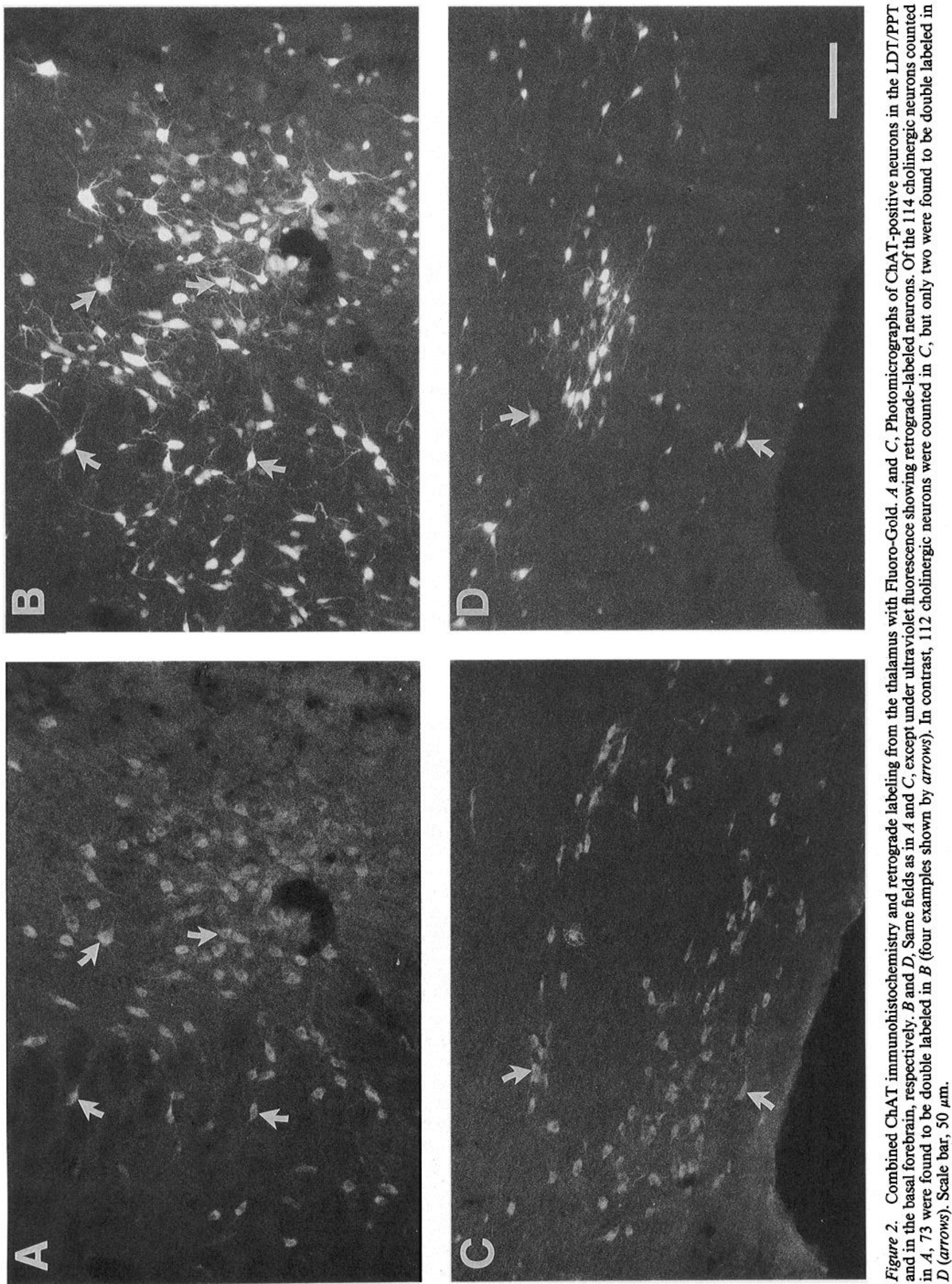
showed that $\mathrm{ACh}$ concentrations varied significantly with behavioral state $[F(2,28)=14.16, p<0.001]$. Further analysis with Scheffe's test showed that ACh concentrations between wake and REM sleep were not significantly different $(t=1.44$, $p=0.173)$, but were significantly higher than that in SWS $(t=$ $5.25, p<0.0001)$.

\section{In vitro recovery of acetylcholine}

The average duration of the REM periods across the 10 rats used in this study was $92.28 \pm 57.87 \mathrm{sec}$. Because the REM periods were short lived, we tested our sample collection technique in vitro in order to determine the extent to which the ACh concentration values obtained for REM sleep were accurate. Microdialysis probes of the type used in this study were connected to polyethylene tubing and perfused with ACSF at a rate of $5 \mu \mathrm{l} / \mathrm{min}$ (see Materials and Methods), The probe was placed in a vial containing normal ACSF and manually transferred to another vial containing $100 \mathrm{nM} \mathrm{ACh}$ for either $30-60 \sec (87 \%$ of the accumulated sample was obtained from intervals of 50 sec or less) or $2 \mathrm{~min}$ intervals. In order to measure total recovery rates, the probe was left in the $100 \mathrm{~nm}$ ACh solution for up to $20 \mathrm{~min}$. Both solutions were maintained at $37^{\circ} \mathrm{C}$. Samples were collected off line, as described i1 Materials and Methods.

Dialysis probes had an in vitro recovery of $22 \pm 3.39 \%(n=$ 4). Collection of 30-60 sec samples reduced the recovery in one probe from $21 \%$ to $12 \%$. Collection of samples from 2 min time periods reduced the recovery in another probe from $17 \%$ to $15 \%$. These results indicate that our in vitro sampling is accurate to $89 \%$ within a 2 min time period, but only $57 \%$ accurate for time periods of $1 \mathrm{~min}$ or less, which suggests that the ACh concentrations measured during REM sleep periods may have been underestimated, as these samples were most likely contaminated with dialysate from SWS periods.

\section{Immunohistochemical verification of cholinergic innervation to the thalamus}

Retrograde tracing with FG resulted in many labeled cells throughout the cortex, hippocampus, basal forebrain, and brainstem. Few neurons were labeled in the medial septum, while a moderate number was found in the more caudal portions of the cholinergic basal nuclear complex, including the horizontal limb of the nucleus of the diagonal band of Broca and in the nucleus basalis of Meynert. Across six rats, a total of 4679 cholinergic cells were counted in the basal forebrain. Of these cells, only 84 or $1.80 \%$ were found to be double labeled. In contrast, 2784 ChAT-positive cells were counted in the LDT and PPT. Of these cells, 1632 or $58.62 \%$ were double labeled (Fig. 2). Therefore, of a total number of 1716 double-labeled neurons, brainstem cholinergic neurons accounted for an average of $94.5 \pm 4.19 \%$, while the basal forebrain accounted for the remaining $5.5 \pm$ $4.19 \%$ (Table 2). These results confirm that the majority of cholinergic inputs to the probe was from the brainstem cholinergic group.

\section{Discussion}

The principle finding of this study is that $\mathrm{ACh}$ release in the thalamus is high during both REM sleep and wake, and significantly lower during SWS. Thalamic ACh concentrations were sensitive to TTX and to calcium-free perfusate, which indicates that the release was due to action potential generation as well as calcium-dependent synaptic transmission. Furthermore, retrograde tracing with FG combined with ChAT immunohisto-
Table 2. Distribution of double-labeled cholinergic neurons

\begin{tabular}{|c|c|c|c|c|c|c|}
\hline \multirow[b]{2}{*}{ Rat No. } & \multicolumn{3}{|c|}{ Basal forebrain } & \multicolumn{3}{|c|}{ LDT/PPT } \\
\hline & $\begin{array}{l}\text { FG+ } \\
\text { ChAT }\end{array}$ & ChAT+ & $(\%)$ & $\begin{array}{l}\mathrm{FG}+ \\
\mathrm{ChAT}\end{array}$ & ChAT+ & $(\%)$ \\
\hline 10 & 21 & 579 & (14) & 124 & 243 & (86) \\
\hline 15 & 21 & 894 & (7) & 264 & 481 & (93) \\
\hline 18 & 2 & 721 & (1) & 228 & 584 & (99) \\
\hline 26 & 6 & 682 & (3) & 221 & 331 & (97) \\
\hline 31 & 13 & 818 & (4) & 325 & 503 & (96) \\
\hline 32 & 21 & 985 & (4) & 470 & 642 & (96) \\
\hline Total & 84 & 4679 & (5) & 1632 & 2784 & (95) \\
\hline
\end{tabular}

For each rat, the number of double-labeled cells (FG + ChAT) versus the total number of cholinergic neurons (ChAT + ) is shown for the basal forebrain and the brainstem (LDT/PPT). Numbers in parentheses show the percentage of all cholinergic neurons projecting to the thalamus. These results show that the majority of inputs to the thalamic probe originated from brainstem cholinergic neurons.

chemistry verified that the majority of cholinergic inputs in the vicinity of the microdialysis probe arose from the mesopontine tegmentum. These results demonstrate unequivocally that mesopontine cholinergic neurons are active during wake.

Based upon the state-dependent activity of serotonergic and noradrenergic neurons (Hobson et al., 1975; McGinty and Harper, 1976; Trulson and Jacobs, 1979; Aston-Jones and Bloom, 1981; Reiner, 1985) and the observation that NA and 5-HT inhibit identified LDT cholinergic neurons in vitro (Luebke et al., 1992; Williams and Reiner, 1993) we hypothesized that mesopontine cholinergic neurons would be profoundly inhibited during wake (Kamondi et al., 1992; Williams and Reiner, 1993). One prediction of this hypothesis is that thalamic $\mathrm{ACh}$ release by mesopontine cholinergic neurons would be much higher during REM sleep than during wake. The present results clearly show that this prediction is in error: thalamic $\mathrm{ACh}$ release during wake and REM sleep is essentially the same. Such a conclusion is in concordance with the results of electrophysiological studies, which suggested that mesopontine cholinergic neurons are active during EEG desynchronized states (El Mansari et al., 1989; Steriade et al., 1990b).

The in vitro recovery rates through the dialysis probes indicated that detection of changes in ACh concentration within short time periods ranging from $30 \mathrm{sec}$ to $2 \mathrm{~min}$ was $57-89 \%$ accurate. This result suggests that the ACh concentrations measured during REM sleep may have been underestimated, as these samples may have been contaminated with dialysate from SWS periods. If this were the case, then it may be possible that $\mathrm{ACh}$ release is higher during REM sleep than wake. This is supported by the observation that while many LDT/PPT neurons were tonically active both during wake and REM sleep, their firing rates were significantly higher in REM sleep as compared to wake (Steriade et al., 1990b). On the other hand, the in vitro sampling error may reflect a time constraint on the rate at which the probes could detect sudden changes in ACh concentration. Behavioral state changes were much more gradual, and as stated earlier, transition periods were discarded. Therefore, it is also possible that the sampling procedure was more accurate in vivo than the in vitro data suggest. In any case, whether extracellular ACh concentrations during REM sleep were underestimated or not, it is clear that mesopontine cholinergic neurons are active during wake as well as REM sleep. This finding is consistent with previous reports showing the state dependence of $\mathrm{ACh}$ release in the cortex (Kanai and Szerb, 1965; Szerb, 1967; Jasper 
and Tessier, 1971) and in the hippocampus (Kametani and Kawamura, 1990), suggesting that this behavioral profile is the same for both mesopontine and basal forebrain cholinergic neurons.

The results of the present study contrast with the current view that ACh release in the mPRF is one mechanism for the induction of REM sleep. This hypothesis is based on the observation that microinjection of the cholinergic agonist carbachol into the $\mathrm{mPRF}$ induces a state that is indistinguishable from REM sleep (George et al., 1964; Baxter et al., 1969; Mitler and Dement, 1974; Amatruda et al., 1975; Gnadt and Pegram, 1986, Quattrochi et al., 1989). In vivo microdialysis of the cat mPRF has shown that extracellular ACh concentrations in the mPRF are very high in both REM sleep (Kodama et al., 1990) and in carbachol-induced REM sleep (Lydic et al., 1991) as compared to wake. Using retrograde tracing combined with ChAT immunohistochemistry, Semba et al. (1990) found that $45-88 \%$ of mesopontine cholinergic neurons projecting to the MPRF also projected to the thalamus. Taken together with the results of the present study, these data suggest that brainstem cholinergic neurons may release ACh differentially with respect to region, ACh being released in the thalamus at equal rates during both wake and REM sleep, but at significantly higher rates in the $\mathrm{mPRF}$ during REM sleep than during wake or SWS. There are several possible mechanisms for this type of ACh release.

First, Semba et al. (1990) also reported distinct populations of brainstem cholinergic neurons that had inputs exclusively either to the thalamus or to the mPRF. This raises the possibility that cholinergic neurons projecting only to the MPRF are selectively active during REM sleep. However, this scenario is unlikely for two reasons. First, Semba et al. (1990) reported that the majority of cholinergic neurons projecting to the mPRF also projected to the thalamus. Second, electrophysiological recordings in vivo have demonstrated that the majority of LDT/PPT neurons are tonically active during wake and REM sleep (El Mansari et al., 1989; Steriade et al., 1990b; Kayama ct al., 1992). Steriade et al. (1990b) did observe a minority of cells that were selectively active during REM sleep, but it is not clear whether these were cholinergic.

A second possible mechanism for differential ACh release is inhibitory axoaxonic inputs onto presynaptic cholinergic terminals in the $\mathrm{MPRF}$, which would suppress $\mathrm{ACh}$ release during wake. Recent anatomical studies have shown that there are neurons in the pontine reticular formation that are immunoreactive for GABA (Brodal et al., 1988) or for glycine (Fort et al., 1993). Microinjection of the $\mathrm{GABA}_{\mathrm{A}}$ agonist muscimol into the rat PRF increases the latency to SWS and to REM sleep as well as the total amount of time spent in wake. Likewise, the GABAergic antagonist bicuculline reduces the latency to SWS and to REM sleep (Camacho-Arroyo et al., 1991). These findings indicate that GABAergic neurotransmission may be involved in the sleep-wake cycle, and presynaptic inhibition of cholinergic terminals in the MPRF may be one aspect of this involvement.

In addition, the rat mPRF receives both serotonergic and (to a lesser extent) noradrenergic inputs arising from the dorsal raphe and locus ceruleus, respectively (Semba, 1993). Semba (1993) hypothesized that waking may be "maintained" in part by the convergence of ACh, NA, and 5-HT onto mPRF neurons, and that REM sleep may be induced upon depolarization of mPRF neurons by $\mathrm{ACh}$ in the absence of noradrenergic and serotonergic input (Ito and McCarley, 1984). Further studies directed at the morphology and cytochemistry of synaptic inputs to the MPRF are needed to address these issues.

In conclusion, the present study has demonstrated that $\mathrm{ACh}$ is relcased in the thalamus at equal rates during wake and REM sleep and much more slowly during SWS. This finding supports the hypothesis that mesopontine cholinergic neurons play an important role in EEG desynchronized states (Steriade et al., 1990b). These data have important implications for the role of ascending as well as descending projections of mesopontine cholinergic neurons in behavioral state control.

\section{References}

Amatruda T, Black D, McKenna T, McCarley RW, Hobson JA (1975) Sleep cycle control and cholinergic mechanisms: differential effects of carbachol injections at pontine brain stem sites. Brain Res 98:501515.

Aston-Jones G, Bloom FE (1981) Activity of norepinephrine-containing locus coeruleus neurons in behaving rats anticipates fluctuations in the sleep-waking cycle. J Neurosci 1:876-886.

Batini C, Moruzzi G, Palestini M, Rossi GF, Zanchetti A (1959) Effects of complete pontine transections on the sleep-wakefulness rhythm: the midpontine pretrigeminal preparation. Arch Ital Biol 97:1-12.

Baxter BL (1969) Induction of both emotional behavior and a novel form of REM sleep by chemical stimulation applied to cat mesencephalon. Exp Neurol 23:220-229.

Brodal P, MihailoffG, Border B, Ottersen OP, Storm-Mathisen J (1988) GABA-containing neurons in the pontine nuclei of rat, cat and monkey. An immunocytochemical study. Neuroscience 25:27-45.

Camacho-Arroyo I, Alvarado R, Manjarrez J, Tapia R (1991) Microinjections of muscimol and bicuculline into the pontinc reticular formation modify the sleep-waking cycle in the rat. Neurosci Lett 129:95-97.

Damsma G, Westerink BHC (1991) A microdialysis and automated on-line analysis approach to study central cholinergic transmission in vivo. In: Microdialysis in the neurosciences (Robinson TE, Justice J, eds), pp 237-252. Amsterdam: Elsevier.

Damsma G, Lammerts van Bueren D, Westerink BHC, Horn AS (1987) Determination of acetylcholine and choline in the femtomole range by range by means of HPLC, a postcolumn enzyme reactor, and electrochemical detection. Chromatographia 24:827-831.

El Mansari M, Sakai K, Jouvet M (1989) Unitary characteristics of presumptive cholinergic tegmental neurons during the sleep-waking cycle in freely moving cats. Exp Brain Res 76:519-529.

Fort P, Luppi P-H, Jouvet M (1993) Glycine-immunoreactive neurones in the cat brainstem reticular formation. Neuroreport 4:11231126.

George R, Haslett WL, Jenden DJ (1964) A cholinergic mechanism in the brainstem reticular formation: induction of paradoxical sleep. Int J Neuropharmacol 3:541-552.

Gnadt JW, Pegram GV (1986) Cholinergic brainstem mechanisms of REM sleep in the rat. Brain Res 384:29-41.

Hallanger A, Wainer BH (1988) Ascending projections from the pedunculopontine tegmental nucleus and the adjacent mesopontine tegmentum in the rat. J Comp Neurol 274:483-515.

Hernandez-Peon R, Chavez-Ibarra G, Morgane PJ, Timo-Iaria C (1963) Limbic cholinergic pathways involved in sleep and emotional behavior. Exp Neurol 8:93-111.

Hobson JA (1965) The effects of chronic brain-stem lesions on cortical and muscular activity during sleep and waking in the cat. Electroencephalogr Clin Neurol 19:41-62.

Hobson JA, McCarley RW, Wyzinski PW (1975) Sleep cycle oscillation: reciprocal discharge by two brainstem neuronal groups. Science 189:55-58.

Ito K, McCarley RW (1984) Alterations in membrane potential and excitability of cat medial pontine reticular formation neurons during changes in naturally occurring sleep-wake states. Brain Res 292:169175.

Jasper HH, Tessier J (1971) Acetylcholine liberation from cerebral cortex during paradoxical (REM) sleep. Science 172:601-602.

Jones BE (1990) Immunohistochemical study of choline acetyltransferase-immunoreactive processes and cells innervating the pontomedullary reticular formation in the rat. J Comp Neurol 295:485514. 
Jones BE, Beaudet A (1987) Distribution of acetylcholine and catecholamine neurons in the cat brain stem: a choline acetyltransferase and tyrosine hydroxylase immunohistochemical study. J Comp Neurol 261:15-32.

Jourdain A, Semba K, Fibiger HC (1989) Basal forebrain and mesopontine tegmental projections to the reticular thalamic nucleus: an axonal collateralization and immunohistochemical study in the rat. Brain Res 505:55-65.

Jouvet M (1962) Recherches sur les structures nerveuses et les mechanismes responsables des differentes phases du sommeil physiologique. Arch Ital Biol 100:125-306.

Jouvet M (1969) Biogenic amines and the states of sleep. Science 163: $32-41$.

Kametani H, Kawamura H (1990) Alterations in acetylcholine release in the rat hippocampus during sleep-wakefulness detected by intracerebral dialysis. Life Sci 47:421-426.

Kamondi A, Williams JA, Hutcheon B, Reiner PB (1992) Membrane properties of mesopontine cholinergic neurons studied with the wholecell patch-clamp technique: implications for behavioral state control. J Neurophysiol 68:1359-1371.

Kanai T, Szerb JC (1965) Mesencephalic reticular activating system and cortical acetylcholine output. Nature 205:80-82.

Karczmar AG, Longo VG, Scotti de Carolis A (1970) A pharmacological model of paradoxical sleep: the role of cholinergic and monoamine systems. Physiol Behav 5:175-182.

Kayama Y, Ohta M, Jodo E (1992) Firing of 'possibly' cholinergic neurons in the rat laterodorsal tegmental nucleus during sleep and wakefulness. Brain Res 569:210-220.

Kodama T, Takahashi Y, Honda Y (1990) Enhancement of acetylcholine release during paradoxical sleep in the dorsal tegmental field of the cat brain stem. Neurosci Lett 114:277-282.

Koyama Y, Kayama Y (1993) Mutual interactions among cholinergic, noradrenergic and serotonergic neurons studied by iontophoresis of these transmitters in rat brainstem nuclei. Neuroscience 55:11171126.

Lindsley DB, Bowden JW, Magoun HW (1949) Effect upon the EEG of acute injury to the brain stem activating system. Electroencephalogr Clin Neurol 1:475-486.

Lindsley DB, Schreiner LH, Knowles WB, Magoun HW (1950) Behavioral and EEG changes following chronic brain stem lesions in the cat. Electroencephalogr Clin Neurol 2:483-498.

Luebke JI, Greene RW, Semba K, Kamondi A, McCarley RW, Reiner PB (1992) Serotonin hyperpolarizes cholinergic low-threshold burst neurons in the rat laterodorsal tegmental nucleus in vitro. Proc Natl Acad Sci USA 89:743-747.

Lydic R, Baghdoyan HA, Lorinc Z (1991) Microdialysis of cat pons reveals enhanced acetylcholine release during state-dependent respiratory depression. Am J Physiol 261:R766-R770.

McCarley RW, Hobson JA (1975) Neuronal excitability modulation over the sleep cycle: a structural and mathematical model. Science 189:58-60.

McGinty DJ, Harper RM (1976) Dorsal raphe neurons: depression of firing during sleep in cats. Brain Res 101:569-575.

Mitani A, Ito K, Hallanger AE, Wainer BH, Kataoka K, McCarley RW (1988) Cholinergic projections from the laterodorsal and pedunculopontine tegmental nuclei to the pontine gigantocellular tegmental field in the cat. Brain Res 451:397-402.

Mitler MM, Dement WC (1974) Cataplectic-like behavior in cats after microinjections of carbachol in pontine reticular formation. Brain Res 68:335-343.

Moruzzi G, Magoun HW (1949) Brain stem reticular formation and activation of the EEG. Electroencephalogr Clin Neurol 1:455-473.

Paxinos G, Watson C (1982) The rat brain in stereotaxic coordinates. New York: Academic.

Quattrochi JJ, Mamelak AN, Madison RD, Macklis JD, Hobson JA (1989) Mapping neuronal inputs to REM sleep induction sites with carbachol-fluorescent microspheres. Science 245:984-986.
Reiner PB (1985) Clonidine inhibits central noradrenergic neurons in unancsthetized cats. Eur J Pharmacol 115:249-257.

Sakai K (1988) Executive mechanisms of paradoxical sleep. Arch Ital Biol 126:239-257.

Satoh K, Fibiger HC (1986) Cholinergic neurons of the laterodorsal tegmental nucleus: efferent and afferent connections. J Comp Neurol 253:277-302.

Satoh K, Armstrong DM, Fibiger HC (1983) A comparison of the distribution of central cholinergic neurons as demonstrated by acetylcholinesterase pharmacohistochemistry and choline acetyltransferase immunohistochemistry. Brain Res Bull 11:693-720.

Semba K (1993) Aminergic and cholinergic afferents to REM sleep induction regions of the pontine reticular formation in the rat. J Comp Neurol 330:543-556.

Semba K, Fibiger HC (1992) Afferent connections of the laterodorsal and the pedunculopontine tegmental nuclei in the rat: a retro- and antero-grade transport and immunohistochemical study. J Comp Neurol 323:387-410.

Semba K, Reiner PB, Fibiger HC (1990) Single cholinergic mesopontine tegmental neurons project to both the pontine reticular formation and the thalamus in the rat. Neuroscience 38:643-654.

Shiromani PJ, Armstrong DM, Gillin JC (1988) Cholinergic neurons from the dorsolateral pons project to the medial pons: a WGA-HRP and choline acetyltransferase immunohistochemical study. Neurosci Lett 95:19-23.

Shouse MN, Siegel JM (1992) Pontine regulation of REM sleep components in cats: integrity of the pedunculopontine tegmentum (PPT) is important for phasic events but unnecessary for atonia during REM sleep. Brain Res 571:50-63.

Shute CCD, Lewis PR (1967) The ascending cholinergic reticular system: neocortical, olfactory and subcortical projections. Brain 90:497520.

Sofroniew MV, Priestly JV, Consolazione A, Eckenstein F, Cuello AC (1985) Cholinergic projections from the midbrain and pons to the thalamus in the rat, identified by combined retrograde tracing and choline acetyltransferase immunohistochemistry. Brain Res 329:213223.

Steriade M, McCarely RW (1990) Brainstem control of wakefulness and sleep. New York: Plenum.

Steriade M, Jones EG, Llinas RR (1990a) Thalamic oscillations and signalling. New York: Wiley.

Steriade M, Datta S, Pare D, Oakson G, Curro Dossi R (1990b) Neuronal activities in brainstem cholinergic nuclei related to tonic activation processes in thalamocortical systems. J Neurosci 10:25412559.

Szerb JC (1967) Cortical acetylcholine release and electroencephalographic arousal. J Physiol (Lond) 192:329-343.

Trulson ME, Jacobs BL (1979) Raphe unit activity in freely moving cats: correlation with level of behavioral arousal. Brain Res 163:135150.

Vincent SR, Reiner PB (1987) The immunohistochemical localization of choline acetyltransferase in the cat brain. Brain Res Bull 18:371415 .

Webster HH, Jones BE (1988) Neurotoxic lesions of the dorsolateral pontomesencephalic tegmentum-cholinergic cell area in the cat. II. Effects upon sleep-waking states. Brain Res 458:285-302.

Westerink BHC, De Vries JB (1988) Characterization of in vivo dopamine release as determined by brain microdialysis after acute and subchronic implantations: methodological aspects. J Neurochem 51: 683-687.

Williams JA, Reiner PB (1993) Noradrenaline hyperpolarizes identified rat mesopontine cholinergic neurons in vitro. J Neurosci 13: 3878-3883.

Woolf NJ, Butcher LL (1986) Cholinergic systems in the rat brain. III. Projections from the pontomesencephalic tegmentum to the thalamus, tectum, basal ganglia and basal forebrain. Brain Res Bull 16: 603-637. 